\title{
Multiatom Resonant Photoemission: Theory and Systematics
}

\author{
F. J. García de Abajo, ${ }^{1,2}$ C. S. Fadley, ${ }^{1,3}$ and M. A. Van Hove ${ }^{1,3}$ \\ ${ }^{1}$ Materials Sciences Division, Lawrence Berkeley National Laboratory, Berkeley, California 94720 \\ ${ }^{2}$ Departamento de CCIA (Facultad de Informática), Donostia International Physics Center, Centro Mixto CSIC-UPV/EHU, \\ San Sebastián, Spain \\ ${ }^{3}$ Department of Physics, University of California, Davis, California 95616
}

(Received 2 November 1998)

\begin{abstract}
A first-principles calculation of the recently discovered interatomic multiatom resonant photoemission (MARPE) effect is presented. In this phenomenon, core photoelectron intensities are enhanced when the photon energy is tuned to a core-level absorption edge of nonidentical neighboring atoms, thus enabling direct determination of near-neighbor atomic identities. Both the multiatom character of MARPE and retardation effects in the photon and electron interactions in the resonant channel are shown to be crucial. Measured peak-intensity enhancements of $40 \%$ in $\mathrm{MnO}$ and spectral shapes similar to the corresponding X-ray absorption profiles are well reproduced by this theory. [S0031-9007(99)09098-5]
\end{abstract}

PACS numbers: 79.60.Bm, 33.60.Fy, 71.15.Rf

In a recent experimental development, interatomic multiatom resonant photoemission (MARPE) has been shown by Kay et al. [1] to provide a new method for determining near-neighbor atomic identities in solids. In the specific example to be considered here, the measured intensity of $\mathrm{O} 1 s$ photoelectrons coming from a single crystal of $\mathrm{MnO}$ exhibits significant enhancements when the photon energy is tuned to the $2 p \rightarrow 3 d$ absorption resonances of $\mathrm{Mn}$. In particular, there is an increase in the $\mathrm{O} 1 s$ peak intensity at the Mn $2 p_{3 / 2}$ edge of $40 \%$ [1]. Similar results have been seen in other metal oxides [1].

In a first discussion of the theory of MARPE [1], the conventional model used to describe the well-known intra-atomic single-atom resonant photoemission (SARPE) [2-5] has been generalized to apply to MARPE. Photoemission is assumed to occur via absorption of a single photon and two different channels that leave the crystal in exactly the same final quantum state, and thus have to be added coherently: the direct emission channel in which an $\mathrm{O} 1 s$ electron absorbs a photon and the resonant emission channel that is assisted by the Mn $2 p \rightarrow \mathrm{Mn} 3 d$ resonance, as illustrated in Fig. 1(a). In the resonant channel, the photon promotes a Mn $2 p$ electron to a Mn $3 d$ state, from which it decays instantaneously to refill the Mn $2 p$ hole, while exciting the $\mathrm{O} 1 s$ electron to the continuum. This decay can also be termed an interatomic autoionization process (AI), and it is thus closely related to the much weaker interatomic Auger processes reported previously [6-8]. Resonant processes must be summed coherently over several Mn neighbors of the emitter [see Fig. 1(b)]. A first estimate from such a theoretical treatment gives an energy-integrated effect of $2.5 \%$, or about 4 times less than the $11 \%$ of experiment [1]. This analysis also suggested that the influence of a given resonating atom should die off as roughly $1 / R^{3}$, if $R$ is the distance from emitter to this atom. However, the model used previously did not permit treating the precise many-electron states and energies involved, and so could not predict the form of the spectral enhancements as a function of energy. This model was also too simplified as to the electromagnetic interactions involved. The present paper thus presents a significantly more quantitative first-principles approach to the theory of MARPE that yields very good agreement with experiment in most respects and permits further assessing the systematics of this new phenomenon. In particular, manyelectron configuration interaction states are used to describe the Mn $2 p \rightarrow$ Mn $3 d$ excitation, retarded effects in
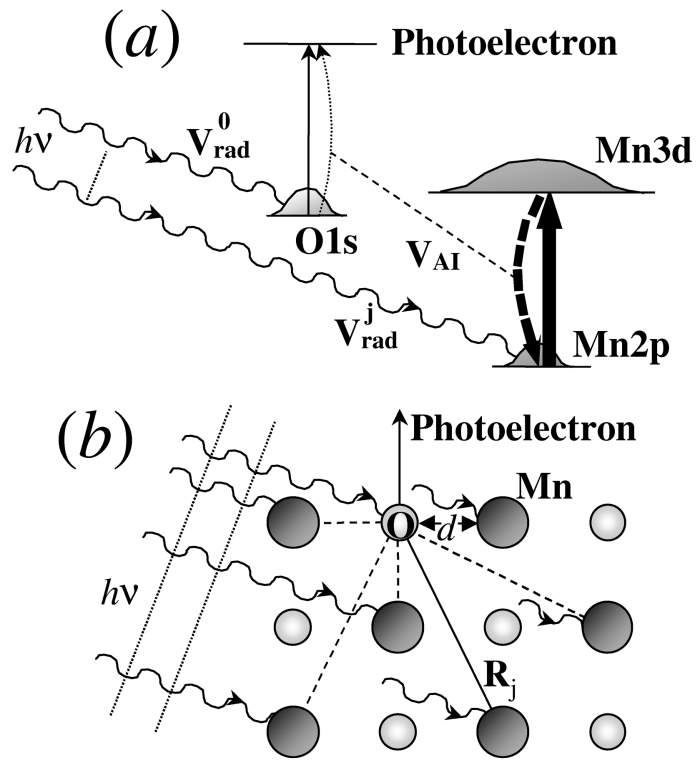

FIG. 1. Schematic representation of multiatom resonant photoemission (MARPE) in MnO. (a) $\mathrm{O} 1 s$ photoemission takes place via two different interfering channels: direct emission (thin solid arrow) and emission assisted by the excitation of $\mathrm{Mn} 2 p \rightarrow \mathrm{Mn} 3 d$ (thick solid arrow) with subsequent interatomic autoionization (broken arrows) due to electron-electron interaction (dashed line). (b) The resonance involves coherent addition over several $\mathrm{Mn}$ sites in the solid situated at $\mathbf{R}_{j}$ with respect to $\mathrm{O}$. 
photon excitation and autoionization are included, and the distance dependence of the effect is assessed in more detail.

We begin with a muffin-tin model for which all finalstate photoelectron scattering is neglected, with such scattering effects to be added later. The intensity of the photoelectron then is

$$
I(\mathbf{k}) \propto\left|\phi_{\mathbf{k}}^{0}(\mathbf{r})\right|^{2} \propto\left|\sum_{l \mu} Y_{l \mu}(\hat{\mathbf{k}}) \mathrm{i}^{l} h_{l}^{(+)}(k r) M_{E l \mu}\right|^{2},
$$

where $\mathbf{k}$ is its wave vector, $\phi_{\mathbf{k}}^{0}(\mathbf{r})$ is its wave function at the detector, $Y_{l \mu}$ is a spherical harmonic, $h_{l}^{(+)}(k r)$ is a spherical Hankel function, and

$$
M_{E l \mu}=\langle E l \mu, \underline{\mathrm{O} 1 s}|T| g\rangle
$$

is the matrix element describing the transition from the many body ground state $|g\rangle$ to the final state with a photoelectron $|E l \mu\rangle$ of energy $E=\hbar^{2} k^{2} / 2 m$ and an $\mathrm{O} 1 s$ hole. The transition matrix $T$ is given by [5]

$$
T=V_{\mathrm{rad}}^{0}+\sum_{j, m} V_{\mathrm{AI}}^{j} \frac{|m, j\rangle\langle m, j|}{\hbar \omega+E_{g}-E_{m}+\mathrm{i} \Gamma_{m} / 2} V_{\mathrm{rad}}^{j},
$$

where $V_{\text {rad }}^{0}$ is the interaction of the radiation with the emitter, $V_{\text {rad }}^{j}$ is this interaction with the resonating atom $j$, $V_{\mathrm{AI}}^{j}$ is the autoionization interaction between the emitter and atom $j, E_{g}$ is the ground state energy, and the sums are over both Mn neighbors $j$ and their intermediate many body states $|m, j\rangle$ of energy $E_{m}$ and width $\Gamma_{m}$.

Relativistic retardation effects are significant in the present context due to a combination of relatively large photon energies $\hbar \omega(\approx 640 \mathrm{eV}$ for $\mathrm{Mn} 2 p \rightarrow \mathrm{Mn} 3 d$ in $\mathrm{MnO}$ ) and the interatomic character of the AI process. For example, the phase change experienced by such a photon which has traveled across the nearest-neighbor O-Mn distance $d=2.23 \AA$ is $\omega d / c \approx 42^{\circ}$ in MnO. As a consequence, the dipole approximation will be valid only within the extension of each atomic orbital, but different phase factors have to be included for different atoms, so that

$$
V_{\mathrm{rad}}^{j}=A \mathbf{r} \cdot \overrightarrow{\boldsymbol{\epsilon}} e^{\mathrm{i} \mathbf{k}_{h \nu} \cdot \mathbf{R}_{j}}=A r \sum_{\lambda} Y_{1 \lambda}(\hat{\mathbf{r}}) \epsilon_{\lambda} e^{\mathrm{i} \mathbf{k}_{h \nu} \cdot \mathbf{R}_{j}},
$$

where $\mathbf{R}_{j}$ is the position of atom $j$ relative to the emitter, $\mathbf{k}_{h \nu}$ is the wave vector of the light, $\overrightarrow{\boldsymbol{\epsilon}}$ is its polarization vector with components $\epsilon_{\lambda}, \mathbf{r}$ is the electron coordinate relative to the atom nucleus, and $A$ is a normalization constant.

Similarly, the autoionization interaction $V_{\mathrm{AI}}^{j}$ requires going beyond the usual $e^{2} / R$ description used in SARPE [5]. The fully relativistic Møller formula used previously in high-energy Auger theory [9] has been adopted for the $\mathrm{AI}$ interaction here, so that

$$
V_{\mathrm{AI}}^{j}=\frac{e^{2}}{\left|\mathbf{R}_{j}+\mathbf{r}_{2}-\mathbf{r}_{1}\right|} e^{\mathrm{i} k_{h \nu}\left|\mathbf{R}_{j}+\mathbf{r}_{2}-\mathbf{r}_{1}\right|}\left(1-k_{h \nu}^{2} \mathbf{r}_{1} \cdot \mathbf{r}_{2}\right)
$$

describes the interaction between electron 1 of coordinate $\mathbf{r}_{1}$ relative to the $\mathrm{O}$ emitter and electron 2 of coordinate $\mathbf{r}_{2}$ relative to the $j$ th resonating $\mathrm{Mn}$ atom. The first term inside the parentheses describes the retarded interaction of the two electron densities, whereas the second $\propto \mathbf{r}_{1} \cdot \mathbf{r}_{2}$ accounts for the retarded interaction of the two currents associated with the transition $[9,10]$.

A multipole expansion of Eq. (5) that should be valid for $r_{1}, r_{2} \ll R_{j}$ (i.e., for atoms other than nearest neighbors) has been performed. Only dipole-dipole terms have been retained, since this analysis shows that higher-order contributions account for less than 5\% of the total interaction. Thus, the effective interaction can finally be reduced to

$$
V_{\mathrm{AI}}^{j} \approx \frac{e^{2} r_{1} r_{2}}{R_{j}^{3}} e^{\mathrm{i} k_{h \nu} R_{j}} \sum_{\mu_{1} \mu_{2}} B_{\mu_{1} \mu_{2}}^{j} Y_{1 \mu_{1}}\left(\hat{\mathbf{r}}_{1}\right) Y_{1 \mu_{2}}^{*}\left(\hat{\mathbf{r}}_{2}\right),
$$

where $B_{\mu_{1} \mu_{2}}^{j}$ are second-order polynomials in $k_{h \nu} \mathbf{R}_{j}$.

Inserting Eqs. (3), (4), and (6) into Eq. (2), and focusing on the $\mathrm{MnO}$ case discussed above, one finds

$$
\begin{aligned}
M_{E l \mu}=A & \langle E 1|r| \mathrm{O} 1 s\rangle \delta_{l, 1} \\
\times \sum_{\lambda} \epsilon_{\lambda}\left\{\left\langle Y_{1 \mu} \mid Y_{1 \lambda} Y_{00}\right\rangle\right. & \\
& \left.-\sum_{\mu_{1} \mu_{2}}\left\langle Y_{1 \mu} \mid Y_{1 \mu_{1}} Y_{00}\right\rangle F_{\mu_{1} \mu_{2}} \alpha_{\mu_{2} \lambda}\right\},
\end{aligned}
$$

where the magnitude of the resonance is dictated by a product of a structure-factor type of sum over Mn sites

$$
F_{\mu_{1} \mu_{2}}=\sum_{j} B_{\mu_{1} \mu_{2}}^{j} \frac{e^{\mathrm{i}\left(k_{h \nu} R_{j}+\mathbf{k}_{h \nu} \cdot \mathbf{R}_{j}\right)}}{R_{j}^{3}}
$$

and the $\mathrm{Mn}^{2+}$ polarizability tensor

$$
\alpha_{\mu_{2} \lambda}=-e^{2} \sum_{m} \frac{\left\langle g\left|r Y_{1 \mu_{2}}^{*}\right| m\right\rangle\left\langle m\left|r Y_{1 \lambda}\right| g\right\rangle}{\hbar \omega+E_{g}-E_{m}+i \Gamma_{m} / 2} .
$$

The dependence of the intermediate states on $j$ has been dropped, since they have been approximated by their uniform bulk form, thus neglecting surface effects. The one-electron radial matrix element of $\mathrm{O} 1 s$ photoemission appears as an overall factor in Eq. (7), so that it is not relevant to the relative magnitude of MARPE.

The polarizability $\alpha_{\mu_{2} \lambda}$ has been obtained using a configuration interaction scheme for a central $\mathrm{Mn}^{2+}$ ion surrounded by six $\mathrm{O}^{2-}$ ions in an octahedral cluster, as used in prior SARPE analyses (see Ref. [5] for further details). Thus, the polarization of nearest-neighbor electronic charge around intermediate states with a Mn $2 p$ hole is accurately included. The widths $\Gamma_{m}$, coming from dominant intra-atomic Coster-Kronig decay of the intermediate 
states, have been taken to be constant $(\approx 0.5 \mathrm{eV})$. Moreover, an average over orientations of Mn magnetic moments has been performed, since the experiment was done above the MnO Néel temperature, and thus, $\alpha_{\mu_{2} \lambda}$ can be approximated by the average quantity $\bar{\alpha} \delta_{\mu_{2} \lambda}$, where $\bar{\alpha}=\left(\alpha_{-1-1}+\alpha_{00}+\alpha_{11}\right) / 3$. The calculated x-ray absorption profile, given by $\operatorname{Im}\{\bar{\alpha}\}$, is in good agreement with experiment [11], as shown in Fig. 2(a). In addition, $\operatorname{Re}\{\bar{\alpha}\}$ shows characteristic sign variations around the resonance, and agrees excellently with a Kronig-Kramers analysis of the experimental absorption coefficient [1].

A small imaginary part has been added to the photon wave vector in both $V_{\text {rad }}^{j}$ and $V_{\mathrm{AI}}^{j}$ to account for inelastic attenuation of the incoming radiation at the Mn $2 p$ edge. This is related to the polarizability via $k_{h \nu}=(1+$ $2 \pi n \bar{\alpha}) \omega / c$ [2], where $n$ is the density of Mn sites, and it yields an attenuation length of $\approx 148 \AA$ at the $\mathrm{Mn} 2 p_{3 / 2}$ resonance maximum in $\mathrm{MnO}$, in reasonable agreement with values inferred from experiment [1].

Multiple scattering effects on the photoelectron have finally been incorporated using an exact Green-function cluster formalism [12]. Using $M_{E l \mu}$ as input, the photoelectron wave function $\phi_{\mathbf{k}}(\mathbf{r})$ is constructed in the far-field $r \rightarrow \infty$ limit as the superposition of spherical outgoing direct and scattered waves like those of Eq. (1) and centered around various atoms (emitter plus scatterers). Clusters of 153 atoms distributed over 5 layers have been used, with an electron inelastic mean free path of $4 \AA$ and an inner potential of $25 \mathrm{eV}[1,13]$. Emitters situated in the first 5 layers have been summed over, with the MARPE summation over $\mathrm{Mn}$ sites in Eq. (8) being performed for the full semiinfinite solid using methods described elsewhere $[14,15]$.

The results of our calculations of the MARPE effect for two different measurement geometries are shown in Figs. 2(b) and 2(c). Plotted is the quantity $I / I_{0}$, where $\mathrm{I}$ is the actual $\mathrm{O} 1 \mathrm{~s}$ intensity and $I_{0}$ is the nonresonant background under it. Curves are shown for both a full sum over all $\mathrm{Mn}$ atoms, and a sum limited to a distance of $\eta d=10 d=22 \AA$ from the emitter. The predicted enhancements follow quite closely the shape of the $\mathrm{x}$-ray absorption profile, showing little evidence of the constructive-destructive interference patterns that are often seen in so-called Fano profiles [2], in agreement with experiment [1]. In general, the limited sum agrees better with experiment as to both peak positions and intensities, suggesting an effectively more rapid convergence with distance in experiment. In magnitude, there is also excellent agreement for the geometry in Fig. 2(b) for an x-ray incidence angle of $20^{\circ}$, although in Fig. 2(c) the principal Mn $2 p_{3 / 2}$ peak is predicted to be too weak by a factor of 2 for an incidence angle of $40^{\circ}$. The possible reasons for this discrepancy are inaccuracies in our treatment of nearest-neighbor effects and the $\mathrm{O} 1 s$ hole state, multiple scattering, and resultant phase averaging and screening of the interaction (much enhanced at the resonance), and neglect of atomic vibrational motion.

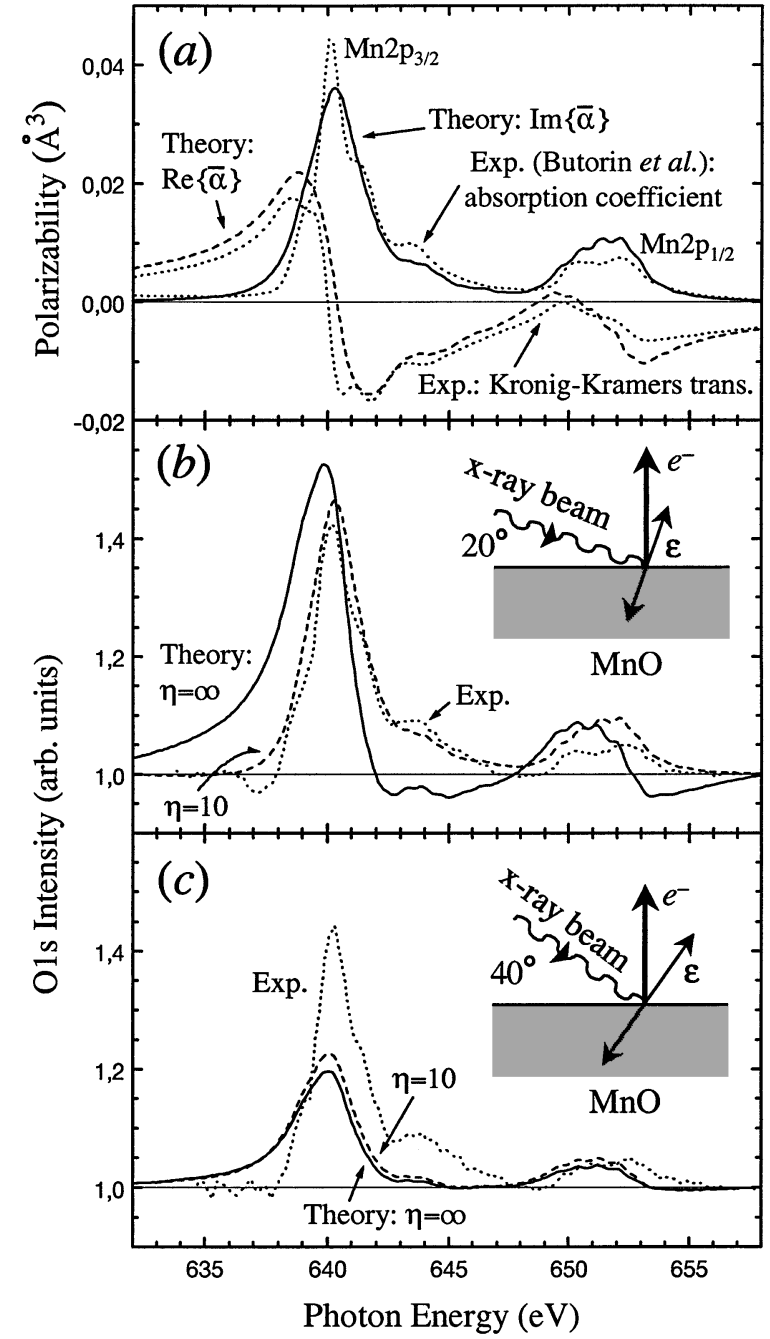

FIG. 2. (a) Real (dashed curve) and imaginary (solid curve) parts of the average polarizability of $\mathrm{Mn}^{2+}$ in $\mathrm{MnO}$, calculated using a configuration interaction scheme [5], as compared with the experimental absorption coefficient [11] and its KronigKramers transformation (dotted curves). (b) Enhancement in the $\mathrm{O} 1 \mathrm{~s}$ photoelectron intensity emitted along the normal of a $\mathrm{MnO}(001)$ surface illuminated with linearly polarized light incident at an angle of $20^{\circ}$ with respect to the surface. The polarization vector $\vec{\epsilon}$ lies in the plane containing [100] and [001]. The experimental result [1] (dotted curve) is compared with theoretical curves for a full sum over all $\mathrm{Mn}$ atoms in each plane (solid curve, $\eta=\infty$ ) and for a sum only out to 10 nearest-neighbor distances (dashed curve, $\eta=10$ ). (c) The same as (b) for an incidence angle of $40^{\circ}$.

Results obtained using different approximations to $V_{\mathrm{AI}}^{j}$ are illustrated in Fig. 3(a). The nonretarded approximation, consisting of setting $k_{h \nu}=0$ in $V_{\text {rad }}^{j}$ and $V_{\mathrm{AI}}^{j}$ [Eqs. (4) and (5)] gives a sizable result (dash-dotted curve), yet significantly lower in magnitude than the measured effect and of different shape. The results obtained using the full Møller expression (solid curve) are very similar to those obtained by keeping only the second term inside the parentheses of Eq. (5) (dashed curve $=$ "current-current" approximation), so that $V_{\mathrm{AI}}^{j} \propto \mathbf{r}_{1} \cdot \mathbf{r}_{2}$, 


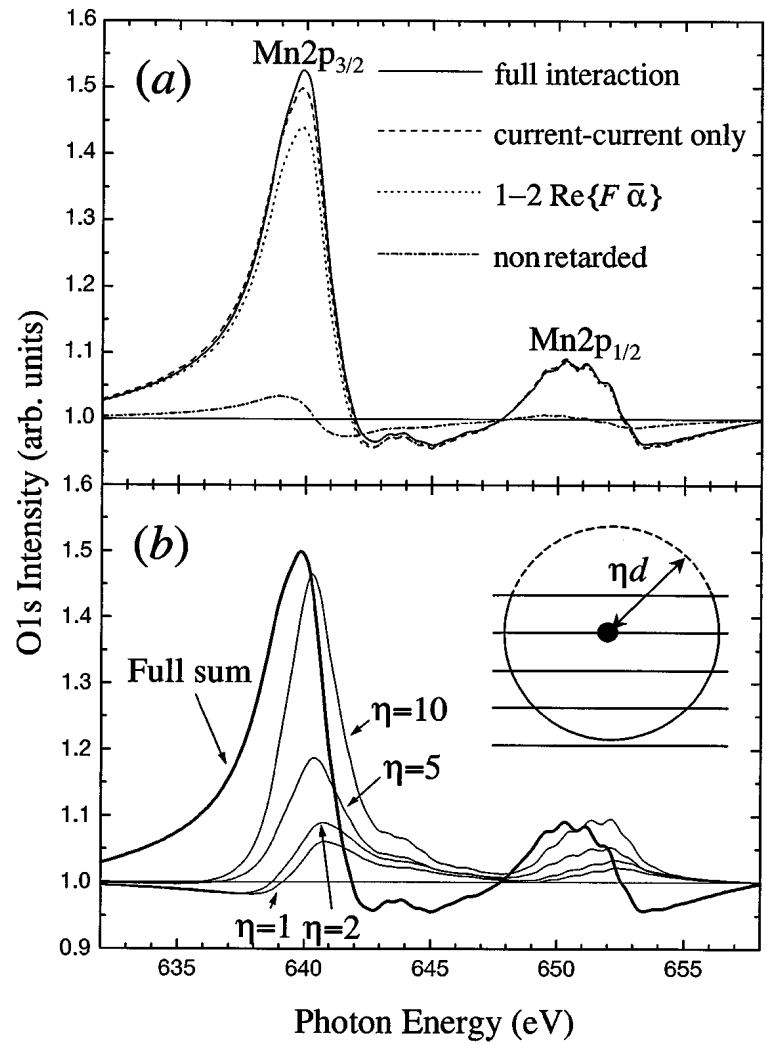

FIG. 3. (a) MARPE enhancement in $\mathrm{O} 1 \mathrm{~s}$ photoemission from $\mathrm{MnO}$ calculated under the same conditions as in Fig. 2(b) and using different approximations to the full theory (solid curve): current-current approximation to the electron-electron interaction, consisting in neglecting the first term inside the parentheses of Eq. (5) (dashed curve); result of neglecting the squared term $|F \bar{\alpha}|^{2}$ in Eq. (10) (dotted curve); and nonretarded approximation (dash-dotted curve). (b) MARPE enhancement of $\mathrm{O} 1 s$ photoemission arising in spheres centered on emitters summed over different layers up to the 5th, under the same conditions as in Fig. 2(b). Only Mn sites closer to the emitter than $\eta d$, where $d$ is the nearest-neighbor distance, are included in the summation over Mn sites in Eq. (8). The result for a full sum over all atoms in each plane is also shown.

and therefore, $F_{\mu_{1} \mu_{2}} \approx F \delta_{\mu_{1} \mu_{2}}$, from which the photoelectron intensity reduces to

$$
I \propto|1-F \bar{\alpha}|^{2}=1-2 \operatorname{Re}\{F \bar{\alpha}\}+|F \bar{\alpha}|^{2} .
$$

Actually, $|F \bar{\alpha}|^{2} \ll 1$, as shown in Fig. 3(a) by the dotted curve, which neglects that term. Moreover, the currentcurrent interaction provides $F$ with a relatively large imaginary part, implying that the MARPE enhancement is actually proportional to $\operatorname{Im}\{\bar{\alpha}\}$, that is, to the $\mathrm{x}$-ray absorption spectrum, in agreement with experiment.

Finally, an estimate of the range of interatomic distances involved in MARPE is shown in Fig. 3(b). The degree of enhancement is shown as a function of a radial cutoff in $R_{j}$ of $\eta$ (in units of the O-Mn nearest-neighbor distance $d$ ), with intensities summed over emitters in the first 5 layers from the surface. That is, only resonating
Mn atoms within $\eta d$ of each emitter are included. Nearest and next-nearest neighbors are seen to be capable of producing a sizable fraction of $\sim 15 \%$ of the effect, with nearly full convergence being reached at about $\eta=10$ (i.e., $22 \AA$ away), which in view of our approximations is probably a realistic estimate of the maximum MARPE range in $\mathrm{MnO}$. The falloff in influence of resonating atoms is found to vary roughly as $1 / R^{2}$ for small distances, and much more rapidly for larger distances.

In summary, the theory of MARPE presented here has permitted explaining both the magnitude of the effect and the shape of the resonance enhancement. It has also been shown that retardation effects are relevant in the understanding of the phenomenon, owing to a combination of relatively large transition energies and interatomic distances. The interatomic autoionization decay is dominated by the interaction between the dipole currents induced in each of the atoms. The range of the effect has also been estimated more quantitatively. Future calculations with more accurate treatment of some of our approximations should improve the description of MARPE and further assist with various applications of this new phenomenon.

Discussions with E. Arenholz, A. Kay, S. Hüfner, S. Mun, and A. Tanaka are gratefully acknowledged. This work was supported by the U.S. Department of Energy, under Contract No. DE-AC03-76SF00098, the University of the Basque Country, and the Spanish Ministerio de Educación y Cultura (Fulbright Grant No. FU-98-22726216).

[1] A. Kay et al., Science 281, 679 (1998).

[2] U. Fano and J. W. Cooper, Rev. Mod. Phys. 40, 441 (1968).

[3] M. O. Krause, T. A. Carlson, and A. Fahlman, Phys. Rev. A 30, 1316 (1984).

[4] L. J. Garvin, E. R. Brown, S. L. Carter, and H.P. Kelly, J. Phys. B 16, L269 (1983).

[5] A. Tanaka and T. Jo, J. Phys. Soc. Jpn. 63, 2788 (1994).

[6] P. H. Citrin, J. Electron. Spectrosc. Relat. Phenom. 5, 273 (1974).

[7] E. Jensen et al., Phys. Rev. B 41, 12468 (1990).

[8] G. K. Wertheim, J. E. Rowe, D. N. E. Buchanan, and P. H. Citrin, Phys. Rev. B 51, 13669 (1995).

[9] N.F. Mott and I. N. Sneddon, Wave Mechanics and Its Applications (Oxford University Press, London, 1948).

[10] J.P. Desclaux, in Relativistic Effects in Atoms, Molecules, and Solids, edited by G.L. Malli (Plenum Press, New York, 1981), pp. 115-143.

[11] S. M. Butorin et al., Phys. Rev. B 54, 4405 (1996).

[12] F. J. García de Abajo (to be published).

[13] B. Hermsmeier et al., Phys. Rev. B 42, 11895 (1990).

[14] K. Kambe, Z. Naturforsch. A 22, 1280 (1968).

[15] J. B. Pendry, Low Energy Electron Diffraction (Academic Press, London, 1974). 